\title{
5G IN RURAL AND LOW-INCOME AREAS: ARE WE READY?
}

\author{
Luca Chiaraviglio, ${ }^{1}$ Nicola Blefari-Melazzi, ${ }^{1,2}$ William Liu, ${ }^{3}$ Jairo A. Gutierrez, ${ }^{3}$ Jaap Van De Beek, ${ }^{4}$ \\ Robert Birke, ${ }^{5}$ Lydia Chen, ${ }^{5}$ Filip Idzikowski, ${ }^{6}$ Daniel Kilper, ${ }^{7}$ Paolo Monti, ${ }^{8}$ Jinsong Wu, ${ }^{9}$ \\ 1) CNIT, Italy, 2) University of Rome Tor Vergata, Italy, 3) AUT, New Zealand, 4) Lulea University of Technology, Sweden, \\ 5) IBM Research, Switzerland, 6) Faculty of Electronics and Telecommunications, Poznan University of Technology, Poland \\ 7) The University of Arizona, USA, 8) KTH Royal Institute of Technology, 9) University of Chile, Chile
}

\begin{abstract}
Current trends in telecommunication networks foresee the adoption of the fifth generation (5G) of wireless networks in the near future. However, a large number of people are living without coverage and connectivity. To face this issue, we consider the possibility of deploying $5 G$ networks in rural and low-income zones. After detailing the current stateof-the-art, we consider the main challenges that need to be faced. Moreover, we define the main pillars to follow in order to deploy $5 G$ networks in such zones, as well as a proposal of a future network architecture.
\end{abstract}

Index Terms - 5G networks, rural and low-income zones, future Internet, global connectivity

\section{INTRODUCTION}

The first experiment on the Arpanet (the father of the current Internet) took place on 29th October 1969, with a simple login message exchanged in a network composed of four nodes. Since then, telecommunication networks have incredibly grown, with distributed architectures connecting billions of users. Today, the Internet is considered as a commodity together with energy, water, and food. According to the recent study presented in [1], the Internet is the world's most powerful engine for social and economic growth, and it needs to be open, secure, trustworthy, and accessible to all. In this scenario, the International Telecommunication Union (ITU) has reported that $69 \%$ of the world population is covered by the third generation $(3 \mathrm{G})$ network [2], which allows users to connect with the Internet. Moreover, the penetration rate of the Internet in North America is above 80\% [3]. However, these numbers are hiding the dark side of telecommunication networks, which is the lack of connectivity and/or coverage experienced by a large number of people, in the non-coverage areas, especially rural and low-income ones. Such zones include both low-density regions, but also towns/cities where the Gross Domestic Product (GDP) is extremely low. To this end, at least two billion people are currently experiencing a complete lack of wireless cellular coverage [4], thus preventing them to connect with the rest of the world.

Given this picture, a natural question is: why are there such differences in connectivity and coverage rates across the world? One initial observation is that the telecommunication networks are widely deployed in urban zones rather than in rural and low-income ones. Specifically, users located in urban areas have the possibility to connect to the Internet by means of $\mathrm{WiFi}$, radio access, fixed access and satellite connections. In this context, telecom manufacturers and researchers are focused on the development of the forthcoming 5G technologies, which will be available by 2020. 5G standards are currently investigated by several organizations around the world, including partnerships (such as 5G Public Private Partnership in Europe, IMT-2020 5G Promotion Group in China, The Fifth Generation Mobile Communications Promotion Forum in Japan, 5G Forum in Korea, and 5G Americas) and international events. These efforts aim to build 5G networks that will dramatically improve the user experience, thanks to a sharp increase in the offered data rates, coupled also with extremely low latency times. In this way, services like very high definition video, tactile Internet, virtual reality and Internet of Things will be made available.

$5 \mathrm{G}$ has several advantages for the spreading of the Internet connectivity (see examples in the white paper of 5G-PPP [5]). Among them, the network introduces a high level of flexibility, which was otherwise not possible with previous technologies. Thanks to this advantage, it is possible to deploy services and network resources where and when they are really needed. In addition, 5G foresees the exploitation of commodity hardware, which opens the way to the development of software solutions implementing networking functions, and potentially decreasing the costs of installing and maintaining devices. Finally, 5G has introduced the concept of "converged solution", where the networks and the services cooperate to deliver high bandwidth and extremely low delay to users.

Even though $5 \mathrm{G}$ introduces several positive aspects, the relevant technologies are "urban" in their nature. Specifically, the high performance requirements are made possible by an extremely rich and complex architecture, including: heterogeneous networks of macro and small cells, fronthaul and backhaul transport networks, small computing nodes deployed close to the users, and large data centres. The current models of telecommunication networks, which are business and profit oriented, suggest that $5 \mathrm{G}$ networks will be mainly deployed in extremely dense urban zones, where the number of subscribers is sufficiently high to compensate the installation and management costs of the $5 \mathrm{G}$ network. On the contrary, rural and low-income zones are less desirable and attractive for operators, since the extremely low 
density (and/or low-income) population does not justify the deployment of $5 \mathrm{G}$ networks.

In this context, the European Union has launched different calls for research proposals in the context of 5G [6]. However, a specific call of $5 \mathrm{G}$ for rural and low-income zones is not included. Rural areas are only indirectly taken into account by the proposals on converged architectures. Such architectures aims at defining a universal network model that can be applied to cities, towns, and small villages. Nevertheless, the associated costs of these networks are still an open issue. In this scenario, the lack of connectivity and coverage for rural and low-income zones is in contrast with the Internet purposes.

In this paper, we take a new view of $5 \mathrm{G}$ networks, by looking at them through the lens of rural and low-income subscribers. In this context, several questions arise: What is the current state-of-the-art in the field of $5 \mathrm{G}$ research in such zones? What are the main challenges that need to be faced? Is it possible to define a holistic 5G architecture explicitly designed for rural and low-income zones? The goal of this paper is to shed light on these issues, and to define future research directions. Specifically, we believe that a $5 \mathrm{G}$ network in rural and low-income zones should be built around the following pillars: i) possibility in deploying cells on board of small Unmanned Aerial Vehicles (UAVs), ii) exploitation of renewable energy sources, iii) reusability of network components and functions, iv) deployment of commodity hardware and $v$ ) reduction of CAPital EXpenditures (CAPEX) and OPerating EXpenditures (OPEX).

The rest of the paper is organized as follow. Section 2 reviews the state-of-the-art. The main challenges are reported in Section 3. Section 4 details our vision. Finally, Section 5 concludes our work.

\section{STATE-OF-THE-ART}

We consider the related work in the following domains: wireless access networks, transport networks, and data centres.

\subsection{Wireless Access Networks}

Future $5 \mathrm{G}$ networks will be characterised by the extensive deployment of small cells [7]. However, this infrastructure requires huge installation and management costs, due to the fact that a large number of sites will be required to be deployed, coupled also with the need of connecting each site to the rest of the network. Currently, one of the great barriers to the development of cellular networks in rural zones is the lack of revenues per square mile [8]. However, even an increase in the competition in the spectrum assignment will not affect the revenue per square mile experienced by operators.

The costs needed to run a wireless network can be divided into two main branches: CAPEX, which are related to the acquisition and the installation of network equipment, and

\footnotetext{
${ }^{1}$ Apart from the connectivity $5 \mathrm{G}$ networks may be exploited also to build the Internet of Things paradigm.
}

OPEX, which instead incur during the management of the network. Focusing on the former, solutions aiming at the deployment of cellular networks with low CAPEX costs are of mandatory importance. These costs can be reduced by decreasing the number of deployed cells per area size. Moreover, authors of [9] clearly show that the exploitation of massive Multiple Input Multiple Output (MIMO) beamforming is able to reduce the network area power consumption by up to $50 \%$ compared to standard cellular networks, while guaranteeing a $10 \mathrm{Mbps}$ cell edge user throughput power. Alternatively, one radical improvement for the reduction of CAPEX costs is the exploitation of new cellular architectures. The Loon project launched by Google [10] aims at providing cellular connectivity by means of balloons naturally moved by the winds of the stratosphere. The same goal is also pursued by the Internet project of Facebook [11], with the main difference that the cells are deployed on board of UAVs flying at high altitude and powered by solar panels.

At the same time, solutions aiming at the reduction of OPEX costs are also necessary. One way to reduce these costs is the exploitation of renewable energy sources. Authors of [12] investigated the adoption of relay nodes powered by renewable sources, showing that the radio resources (in terms of Resource Blocks and transmission power) can be efficiently allocated in order to maximize the user data rates. Moreover, renewable sources can be also exploited to power macro cells. In this context, solar powered cells are being deployed to provide connectivity, mainly in some zones of Africa and Asia [13]. Even though the size of the renewable energy power systems is still an issue [14], the cost of a solar powered cell is comparable with a cell connected to the grid, thus much less than exploiting power coming from expensive diesel generators. In addition, the OPEX costs can be decreased by reducing the power consumption of cells [15]. This is made possible by the exploitation of different power states (such as full power and low power) which are applied over time, by taking advantage of the traffic variability. Finally, another way to reduce the OPEX is the adoption of virtual network elements, for example by means of Cloud Radio Access Networks (C-RAN) [16].

\subsection{Transport Network}

With the latest $5 \mathrm{G}$ requirements of individual user peak access rates between 1 and $50 \mathrm{~Gb} / \mathrm{s}$ [5], fibre connections become essential and therefore fibre networks will need to reach rural and under-developed areas, where it is important to give priority to other characteristics, i.e., low cost/power; resilience to power disruptions, and low maintenance requirements [17].

When looking at today's fronthaul-backhaul infrastructures, it can be noticed that radio systems employ a "remote radio head" strategy in which the Radio Frequency (RF) signal is sampled and immediately converted to an optical signal for transmission to the Base Band Unit (BBU) processing the base band. These units can be placed at the bottom of the tower or centralized in BBU hotels that can be quite far away 
from the antenna. In this case however, the radio processing delay requirements become the limitation. In LTE the UpLink Hybrid Automatic Repeat reQuest (UL-HARQ) has a $4 \mathrm{~ms}$ response time requirement that, given the processing time requirements, translates into a maximum fibre distance of $20 \mathrm{~km}$. A small amount of additional headroom can significantly increase the optical reach. This would open the possibility to distribute the low-cost, low-power, high-reliability radio heads across an area extending $20 \mathrm{~km}$ from a centralized processing location, where higher reliability, security, and greater efficiency may be possible [18].

When considering core transport networks (i.e., the ones used to move data from/to access networks), renewable energy such as solar will likely be important for many rural areas with limited power availability. Their drawbacks is mainly in their lack of reliability. In this context, several studies have looked at the operation of optical systems in the presence of a variable and renewable energy sources (see e.g., [19]).

Another way to reduce the cost and improve the resource efficiency in a transport network is to introduce advanced network functionalities (i.e., dynamic resource sharing and Network Function Virtualization (NFV)) [20]. This allows for example the allocation of resources on-demand to support specific transport needs that may vary over time, without the need of manually setting up the devices. Dynamic resource sharing is based on the intuition that the same transport resource can be dynamically shared over time for different transport purposes. NFV provides flexibility by dynamically placing network functions in different locations depending on the specific need of a service, e.g., close to the users to exploit traffic locality. Examples of network functions that can be virtualized include for instance Evolved Packet Core (EPC) functionalities for local breakout, virtualization of packet aggregation capabilities, and virtualization of computing and storing functionalities, e.g., network caching.

These advanced functionalities required the presence of distributed (possibly small) Data Centre (DC). Therefore, the possibility to have low-power low-cost DC solution is crucial. In this respect optics can help again [21]. Small form factor optics for DCs use minimal electronics and trade performance for low cost. Long reach transceivers are available up to $100 \mathrm{~Gb} / \mathrm{s}$ and $80 \mathrm{~km}$ reach. Continued progress in reducing the cost and form factor of these optics will be important for applications in rural and low-income areas. Integrated photonics is a promising approach to doing this [22]. In particular, silicon photonics integrates multiple photonic devices on silicon chips, which are often Complementary Metal Oxide Semiconductor (CMOS) compatible, with the potential for optical devices to share the same cost benefits of high-volume micro-electronics. Recently, singlechip Wavelength Division Multiplexing (WDM) chips were manufactured with $500 \mathrm{~Gb} / \mathrm{s}$ aggregate data rate and as many as 1700 devices [23]. This Indium Phosphide (InP) based chip was designed for long-haul links. Intense research and development are currently underway for silicon photonic transceivers that can be manufactured in high volume and low cost [24].

\subsection{Data Centres}

Data centres are proliferating worldwide, but mostly in urban areas and developed countries, thus increasing the global digital divide. ${ }^{2}$ The reason is that the conditions in rural and developing countries often exacerbate the challenges for operating data centres in addition to prohibitive acquisition costs. A part from the previously discussed connectivity problems, energy provisioning raises several challenges. Specifically, in developing countries electrification is often not complete and especially rural areas lack access to the electric grid. To cope with energy variability and security, research on demand-response and emergency demand-response [25] tries to optimize the data centre operation based on the energy costs and availability. For this, they exploited the large body of research which went into power consumption flexibility at data centres via capacity right-sizing [26], load shifting over time [27] and across geographies [28].

To overcome the lack of grid access and to green the impact of data centres as well as to drive down the CAPEX costs, data centres are scaled down in size [29], powered via renewable energies [30] and rely on micro-servers with better work done per joule and work done per dollar ratios [31].

To reduce the energy consumption and OPEX costs, research tries to optimize the three major pillars in data centre: IT, cooling and power (see e.g., [33]). The upcoming 5G also promotes the use of softwarization and virtualization as means to drive up efficiency [34], especially via cloud-based radio access networks [35].

\section{CHALLENGES}

We first review the challenges related to the exploitation of $5 \mathrm{G}$ technologies iin rural and low-income zones the first step, and then we consider the socio-economic aspects that need also to be taken into account.

5G Technology Challenges The application of 5G in rural and low-income areas is challenged by the peculiar features of such scenarios. Tab. 1 reports a comparison between a classical 5G urban scenario [5] against 5G rural and lowincome ones ${ }^{3}$ (whose requirements may be inferred from [36]). In contrast to $5 \mathrm{G}$ urban's most advanced Internet services such as High-Definition (HD) streaming, tactile Internet and Internet of Things, many rural and low-income regions are still disconnected from the rest of the world and the lack of Internet coverage is the most critical problem to be tackled. The state of several essential services such as e-Learning and e-Health have remained substandard. Therefore, the infrastructure is required to support the

\footnotetext{
${ }^{2}$ Data centres requirements may be different than the ones of telecommunication networks, which are mainly based on central offices.

${ }^{3}$ Low-income areas include both low density regions in terms of populations as well as town and cities.
} 
Table 1. Comparison of a classical 5G Urban Scenario with Rural and Low-income Ones

\begin{tabular}{|l|l|l|l|}
\hline & 5G Urban Scenario & 5G Rural Scenario & 5G Low-income Scenario \\
\hline Service Type & $\begin{array}{l}\text { HD Video, HD Streaming, Tactile } \\
\text { Internet, IoT }\end{array}$ & $\begin{array}{l}\text { HD Video, Emergency Service, e- } \\
\text { Health, e-Learning }\end{array}$ & $\begin{array}{l}\text { Basic Connectivity, Emergency } \\
\text { Service, Delay Tolerant, e-Health, } \\
\text { e-Learning }\end{array}$ \\
\hline $\begin{array}{l}\text { Network Con- } \\
\text { straints }\end{array}$ & $\begin{array}{l}\text { Maximize Bandwidth, Minimize } \\
\text { Delay, Coverage }\end{array}$ & Coverage, Guaranteed Bandwidth & Coverage \\
\hline Energy Sources & Power Grid & Power Grid, Renewable Sources & $\begin{array}{l}\text { Unreliable Power Grid and/or Re- } \\
\text { newable Sources }\end{array}$ \\
\hline $\begin{array}{l}\text { Network Cost } \\
\text { from the User } \\
\text { Side }\end{array}$ & Pay per bandwidth & Same as standard urban users & Low \\
\hline $\begin{array}{l}\text { Business } \\
\text { Model }\end{array}$ & Return on Investment & Subsidized by the government & Subsidized by the government \\
\hline $\begin{array}{l}\text { Required Net- } \\
\text { work Flexibil- } \\
\text { ity }\end{array}$ & High & High & High \\
\hline User Mobility & $\begin{array}{l}\text { Pedestrian, Vehicular, High Speed } \\
\text { Vehicular }\end{array}$ & Pedestrian, Vehicular & Pedestrian, Low Speed Vehicular \\
\hline
\end{tabular}

appropriate set of applications to guarantee these essential services according to their different network constraints such as bandwidth requirements and link reliability. While for urban zones it is important to maximize the bandwidth and minimize the delay, in rural areas it is essential to guarantee a given minimum amount of bandwidth to users (e.g., the one required to deliver video services - but not high definition). Additionally, in rural and low-income zones it is even more important to guarantee basic coverage rather than high bandwidth services. In addition, another aspect that can not be neglected is that in rural and low-income zone the power grid may be not always available and/or unreliable, thus suggesting that renewable sources (such as the sun and the wind) should be exploited. Moreover, the cost of the network from the user side should be kept as low as possible, keeping also in mind that in low-income areas the users should pay much less for an Internet connection compared to the urban regions. This also inevitably influences the associated business models in such zones, which can not be based on the classical Return On Investment (ROI), but rather on the fact that the Internet is a primary need, that should be provided, e.g., by the government rather than private operators. ${ }^{4}$ Moreover, the network has to be flexible in all scenarios. For example, in rural and low-income zone the network has, e.g., to deal with the scarcity of electricity, as well as to wisely manage the network resources in order to guarantee coverage. Finally, the user mobility has to be always taken into account. Specifically, rural and low-income zones are characterised by relatively lower mobility compared to urban ones. ${ }^{5}$ This feature may also have an influence on the design

\footnotetext{
${ }^{4}$ Private operators will not invest the cost of an infrastructure in such zones, due to the low return on investment. Therefore, the government should be either invest on the deployment of a minimum infrastructure or even becoming a network provider.

${ }^{5}$ In urban zones users may travel on high speed trains across the city. In rural zones users are more fixed, e.g., they are located close to their houses. In low-income zones users may be traveling by foot or by means of lowspeed vehicles.
}

of the network in such zones.

Socio-Economic Challenges In addition to these aspects, we would like to stress that networking alone is not sufficient to enable effective usage of its applications and services for a sustainable development. Other socio-economic challenges are also to be taken into account. First, affordability is to be considered in the networking development if the citizens want to use it effectively to achieve better living conditions. The cost of broadband connectivity is still higher than the average income in developing countries. Therefore, it can be a financial barrier for the poor citizens and communities from benefiting from networking. Second, it is also crucial to consider how relevant are applications and services provided. Their users, especially the low-income people, need applications that are necessary to their primary development needs such as the basic instructions they can understand, accessible through devices and services that they can afford and use conveniently. Third, human capacity is as critical as applications. Users need knowledge and skills to fully benefit from networking-enabled services, including ICT-based skills in the areas such as computer networking, web and basic applications development and elementary network security are essential in all societies. Governments and policy-makers need to understand the technical aspects of networking and their services, as well as the interplay between technological and public policy domains. Finally, the impact of the networking on environment is also critical. The networking can drive energy efficiency, smart systems and services to enable more productivities. However, networking is to be also a growing source of material consumption and greenhouse gas (GHG) emissions. This negative impact will increase as networking become more and more widespread.

\section{OUR VISION}

Given the aforementioned challenges, we first define the main pillars which, we believe, are essential for the design 
and management of $5 \mathrm{G}$ networks in rural and low-income zones. Our architecture is driven by the fact that the Internet connectivity is a primary need, that may be provided by the government or government-based entities rather than private ones. This alleviates the need of always guaranteeing a ROI. However, this process should be coupled with the reduction of the costs needed to deploy and manage the network. In the following, we sketch the proposed architecture.

\subsection{Main Pillars}

Converged Solution We believe that the services and the networks should be managed in a converged way, following a trend that is currently emerging in $5 \mathrm{G}$ architectures [37]. In our scenario, the last mile of the network should be orchestrated in conjunction with the metro and core one, without a complete separation between them, in contrast to current networks. Additionally, the services are not running on "top" of the network, but they are lying at the same level of the network components. More in depth, the network provider is also acting as service provider, and different computing components (like storage, local DC, caches) are installed on most network devices both in metro/core and access segments. As a results, there is not a strict separation between the different parts of the network, thus enabling a global optimization of the services and the network. This design choice is also justified by the fact that rural and low-income zones will be likely managed by government-based entities, which will then have the highest flexibility to deploy the services and the different components across the full network.

Reusability of Network Components We believe that another of the main pillars will be the possibility to completely virtualize most of the network components by means of virtual functions that are shared among devices and managed by a centralized entity. This trend is in line with current efforts in the broad area of softwarization, which aims to bring the network and computing functionalities from the hardware space to the software environment. Even though the performance of virtualized network elements is still lower than fully hardware-based components, this constraint is less stringent in rural and low-income zones compared to urban ones, thanks to the fact that the strict requirements in terms of high bandwidth and extremely low delays can be relaxed in such contexts. Therefore, by letting each element to be composed of a set of virtual elements, it is possible to reuse the same network components across different physical devices. This feature triggers the possibility to implement smart resource allocation policies in order to move the network and the computing capacity where it is really necessary or where the sources of energy are currently available. Moreover, with this capability the functionalities of network devices can be directly modified by software upgrades, without the need of changing the underlying hardware, which otherwise may be an expensive operation. Finally, thanks to the fact that the devices are split in software components, it is possible to integrate several functions in a single device, e.g., networking and computing functions.
Exploitation of Commodity Hardware In traditional networks, where most of functionalities are hardware-coded, there is a clear lack of flexibility. Each device (either a Base Station, a router, or a computing node) is composed of proprietary hardware, which can be hardly managed in a converged solution where all the network components and the services need to be controlled in a flexible way. In our vision, the network components are softwarized, and therefore it is possible to adopt general purpose hardware for most of devices. Apart from the flexibility aspects, the exploitation of commodity hardware has additionally two main advantages: i) the costs of deploying the network can be cheaper compared to high performance hardware-coded devices, and ii) since the hardware is the same for most of devices also the operating expenses can be reduced (e.g., in terms of failure costs). Clearly, the use of softwarized functions and commodity devices have an impact on the performance (e.g., bandwidth and delay), which should be always taken into account.

Solar-Powered Energy-Efficient Devices Since the power grid is assumed to be not so widespread in rural zones and not present or unreliable in low-income zones, a clear pulse will be the large exploitation of renewable energies to power the physical devices. Among the available renewable sources, one of the most promising is the sun. Moreover, since most low-income zones are located in the regions of the earth receiving the largest irradiation from this energy source, we expect that a large exploitation of solar panels will be a viable approach. However, even though the solar power can be predicted over time (thanks to weather forecasts), it is obviously not always available (e.g., during night or bad weather conditions). In addition, the size of the solar panels is still an issue for the installation costs. Therefore, the elements of a network have to carefully manage their energy consumption, by implementing, e.g., smart energy saving policies to reduce the amount of power requested. These policies will integrate both the knowledge of the power available and the variation of traffic experienced in the network over time.

Unmanned Aerial Vehicles and Advanced Radio Techniques In order to limit the costs for installing and managing the devices, the number of nodes devoted to the last segment of the network should be reduced as much as possible. Focusing on wireless elements, we foresee the exploitation of different technologies. First of all, thanks to the recent advances in Unmanned Aerial Vehicles (UAVs), the radio elements may be mounted on top of them. For example, it would be possible to move the radio nodes in order to follow the (few) users in a rural network, thus preventing them from the lack of connectivity. In addition, radio elements can be deployed at higher altitudes, in order to exploit two different features: i) the winds that can move such devices in the atmosphere, and ii) the fact that most users will experience Line Of Sight (LOS) conditions, which will decrease the propagation loss compared to classical Non Line of Sight (NLOS) conditions. In case of rural zones in which the number of users is not so low compared to the previous case, the emerging technology of massive antenna arrays allows 


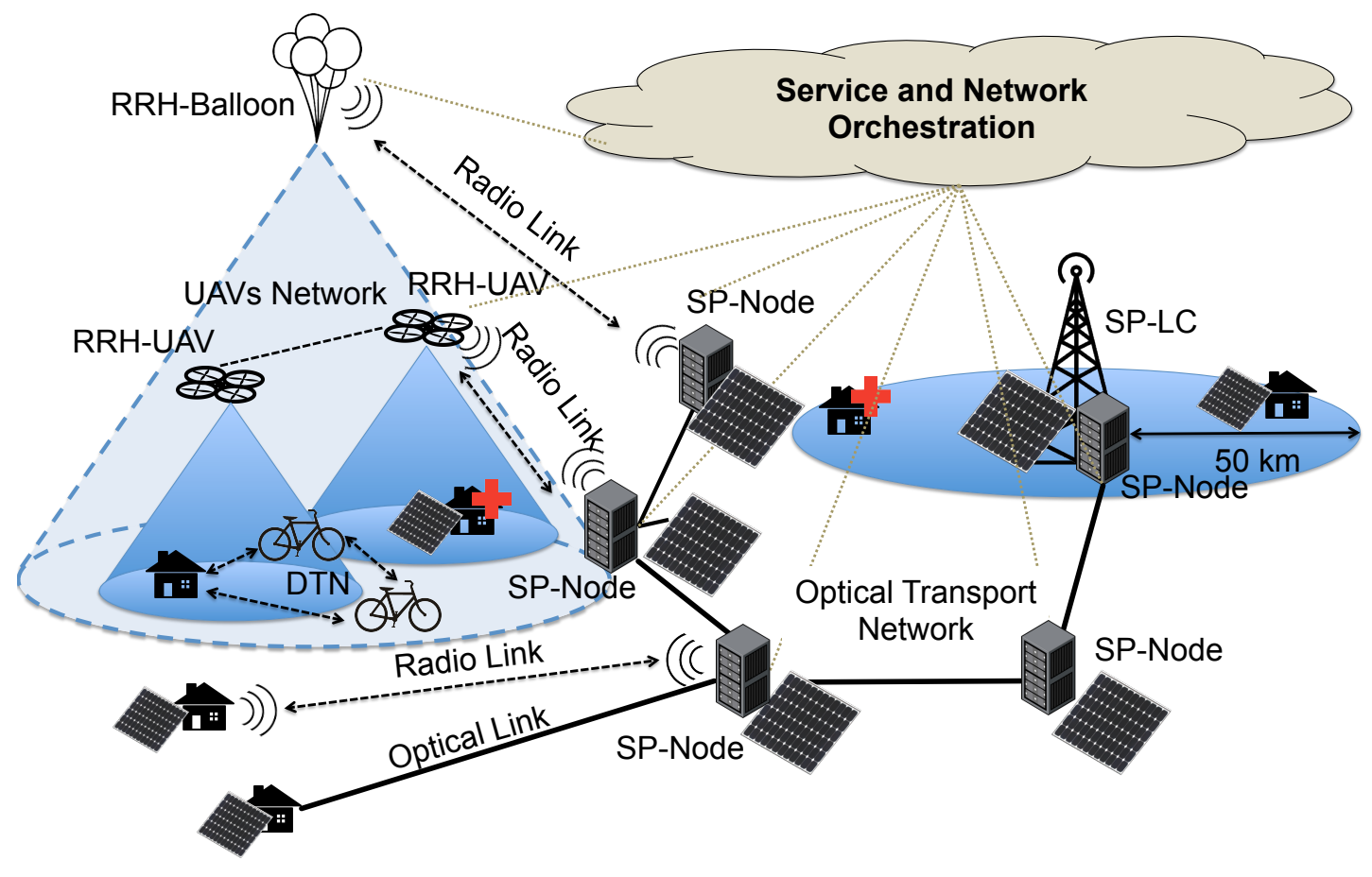

Fig. 1. Vision of a 5G network for rural and low-income zones. $(\mathrm{SP}=$ solar powered, LC $=$ Large Cell, RRH = Remote Radio Head, UAV = Unmanned Aerial Vehicle, DTN = Delay Tolerant Network, NODE $=$ Flexible component that can act as micro server, BBU, SDN switch and optical router).

the deployment of radio elements covering ultra-large cell sizes (over $50 \mathrm{~km}$ ). It is widely acknowledged in academia and industry that deployment of very large antenna arrays at the base station side will form a key component of the $5 \mathrm{G}$ radio standards that very recently have started to be addressed by 3GPP. Technologies in academia referred to as massive MIMO are in the heart of the urban, capacity-driven $5 \mathrm{G}$ use cases and scenarios. Moreover, the energy-efficiency of these very large antenna arrays is also promising for the noise-limited, low-load rural and remote coverage scenarios. ${ }^{6}$ The large arrays can accomplish a beamforming gain that will beneficially affect the link budgets of large macrocells. The pencil-sharp radiobeams produced by these base stations allow to increase the cell radius without compromising implementation complexity, cost-efficiency or energy consumption. Finally, we foresee the exploitation of new ultra-lean radio-protocols, which will be developed with the explicit goal in reducing the transmission overhead of the radio nodes and improving their energy efficiency [38].

\subsection{Overall Architecture}

Our vision aims to develop a comprehensive low-cost connectivity architecture that can efficiently support a widerange of services and applications. Fig. 1 reports the proposed architecture. Focusing first on the access part of the network, we foresee the exploitation of different technolo-

\footnotetext{
${ }^{6} \mathrm{We}$ refer the reader to the Facebook project ARIES: https: //code.facebook.com/posts/1072680049445290/ , lastaccessedon1stJuly2016.
}

gies. Specifically, for rural zones with a limited number of users, coverage and capacity may be provided by RRH mounted on top of UAV (RRH-UAV). These devices can provide flexible coverage over a territory, by considering only the zones where the users are located. Moreover, RRHUAVs can be exploited when the capacity of the network is needed (e.g., during the day). Each RRH-UAV establish communication with the other RRH-UAVs flying in the same zone. The goal is then to compute the UAV trajectories in order to optimize the coverage of the zone where the users are located. In addition, the RRH-UAV will establish a radio link with a BBU mounted in selected Solar-Powered (SP) Nodes 7. The challenge here will be to develop smart solutions to reduce the amount of information exchanged between the RRH-UAVs and the SP-Node with the BBU. Notice also that he UAVs can be recharged by power stations fed by solar panels. In addition to this, RRHs are also mounted on top of balloons. These elements are continuously flying in the atmosphere in order to provide basic coverage and emergency services. Finally, each community connected to such an infrastructure may develop Delay Tolerant Networks (DTNs) to further spread the information by means of low-velocity vehicles (i.e., mainly bicycles). As second alternative to provide wireless access connectivity, we foresee the exploitation of Large Cells (LCs), with coverage radius in the order of 50 $\mathrm{km}$. Such cells can be spread in low-income areas where the users requirements are low in terms of bandwidth and delays. LCs are also powered by Solar Panels (SPs), since the power

\footnotetext{
${ }^{7}$ This solution has to be evaluated w.r.t. the LTE UL-HARQ latency constraint.
} 
grid is assumed to be not present or unreliable. The main issue in this scenario will be to deploy efficient solutions to limit the amount of required power in the uplink. Finally, we foresee the exploitation of direct optical connections and dedicated radio links to selected locations (e.g., places where the bandwidth requirements are higher).

Looking then at the other network solutions, we foresee the exploitation of flexible, efficient, low-cost, low-power nodes (SP-Nodes in the figure). Such devices, which will be solar powered, will virtualize different functionalities, including radio, computing, and transport ones. Each functionality can be activated/deactivated depending on where the node is located (in the transport part of the network or in the access one) and when it is needed. Moreover, the interconnection between the SP-Nodes will be realized by means of low-cost and low-power optical connections, which will implement the state-of-the-art features to increase the efficiency of the entire architecture.

Finally, the architecture will be controlled by a centralized orchestrator, which will jointly manage the network and the computing resources. For example, during period of high traffic the computing resources will be moved in the SPNodes close to users, while the opposite will be realized during low traffic periods. At the same time, coordination of UAVs, as well as decrease/increase of LC coverage will be done in accordance to the users variation over time. Finally, the orchestrator will perform this allocation of resources also taking into account the variation of power available from the SP.

\section{CONCLUSIONS AND FUTURE WORK}

We have focused on the problem of providing $5 \mathrm{G}$ services in rural and low-income areas. After a deep dive into the current state-of-the-art, we considered the main challenges that need to be faced for a full exploitation of $5 \mathrm{G}$ in such areas. In order to achieve this goal, we discussed a number of architectural features, including: the adoption of a converged solution, the reusability of network components, the exploitation of commodity hardware, the deployment of solar powered energy-efficient devices, UAVs and advanced radio techniques. Additionally, we proposed a reference architecture.

As future work, we plan a number of research activities. First of all, a detailed characterization of requirements of users is mandatory in both rural and low-income zone. This task would then drive the definition of more detailed technology solutions targeted to each specific context. In addition, we plan to study the radio issues that may emerge. For example, a large cell may provide coverage over a vast area. However, the uplink budget may be constrained by the user power and therefore there is an issue when the terminal is far from the macro cell, since a high amount of uplink power would be required. Moreover, different functional splits aiming to reduce the amount of data transferred between the RRH and the BBU will be also investigated. In addition, we plan to tackle the problem of computing the optimal UAVs trajec- tory to serve a set of users in a rural area. Finally, we plan also to analyse the proposed solutions in terms of CAPEX and OPEX, as well as properly dimensioning the solar panels needed to run the networks and services.

\section{ACKNOWLEDGMENTS}

This work has received funding from the project EU Horizon 2020 Superfluidity (grant agreement No. 671566) and the Polish National Science Center (decision DEC2014/12/S/ST7/00415). We would like to thank the anonymous reviewers for their suggestions.

\section{REFERENCES}

[1] "The future of the Internet is at risk say global web experts," http: //www.ecs.soton.ac.uk/news/4890.

[2] "International Telecommunication Union (ITU), ICT Facts and Figures the world in 2015," http: / /www. itu.int/ en/ITU-D/Statistics/Pages/facts/.

[3] "World internet penetration rates," http://www. internetworldstats.com/stats.htm.

[4] Mats Eriksson and Jaap Van de Beek, "Is Anyone Out There? 5G, Rural Coverage, and the Next One Billion," in Comsoc Technology News, 2015.

[5] "5G-PPP White Papers," https://5g-ppp.eu/ white-papers/.

[6] Arturo Atzcorra, "Trend in EU Research for 5G Networks," https://ans.disi.unitn.it/inw2015/ presentations/s4-1-azcorra-5g.pdf.

[7] Cheng-Xiang Wang, Fourat Haider, Xiqi Gao, Xiao-Hu You, Yang Yang, Dongfeng Yuan, Hadi M Aggoune, Harald Haas, Simon Fletcher, and Erol Hepsaydir, "Cellular architecture and key technologies for 5G wireless communication networks," IEEE Communications Magazine, vol. 52, no. 2, pp. 122-130, 2014.

[8] Anna-Maria Kovacs, "Regulation in financial translation: Will the incentive auction increase mobile-broadband competition in rural america?," Tech. Rep., Georgetown University McDonough School of Business, May 2014, http://www.gcbpp.org/files/EPV/Kovacs_ Spectrum_Auctions_Rural_5.1.2014.pdf?utm_ source=Anna-Maria+EPV\&utm_campaign=EVP+ 5\%2F1\&utm_medium=email.

[9] Paal Frenger, Magnus Olsson, and Erik Eriksson, "Radio network energy performance of massive mimo beamforming systems," in Proceedings of the IEEE 25th Annual International Symposium on Personal, Indoor, and Mobile Radio Communication (PIMRC). Sept. 2014, pp. 1289-1293, IEEE.

[10] "Google Loon Project," https://www.google.com/ loon/.

[11] "Facebook Internet Project," https://info. internet.org/.

[12] Sobia Jangsher, Haojie Zhou, Victor O.K. Li, and Ka-Cheong Leung, "Joint allocation of resource blocks, power, and energy-harvesting relays in cellular networks," Tech. Rep. 3, Mar. 2015. 
[13] Vinay Chamola and Biplab Sikdar, "Solar powered cellular base stations: current scenario, issues and proposed solutions," IEEE Communications Magazine, vol. 54, no. 5, pp. 108-114, 2016.

[14] Marco Ajmone Marsan, Giuseppina Bucalo, Alfonso Di Caro, Michela Meo, and Yi Zhang, "Towards zero grid electricity networking: Powering bss with renewable energy sources," in 2013 IEEE international conference on communications workshops (ICC). IEEE, 2013, pp. 596-601.

[15] Ziaul Hasan, Hamidreza Boostanimehr, and Vijay K Bhargava, "Green cellular networks: A survey, some research issues and challenges," IEEE Communications surveys \& tutorials, vol. 13, no. 4, pp. 524-540, 2011.

[16] Peter Rost, Carlos J Bernardos, Antonio De Domenico, Marco Di Girolamo, Massinissa Lalam, Andreas Maeder, Dario Sabella, and Dirk Wübben, "Cloud technologies for flexible $5 \mathrm{~g}$ radio access networks," IEEE Communications Magazine, vol. 52, no. 5, pp. 68-76, 2014.

[17] Daniel C. Kilper and Houman Rastegarfar, "Energy challenges in optical access and aggregation networks," Philosophical Transactions of The Royal Society A, vol. 374, no. 2062, Mar. 2016.

[18] Seamas McGettrick, Frank Slyne, Nattapong Kitsuwan, David B. Payne, and Marco Ruffini, "Experimental end-toend demonstration of shared N:1 dual homed protection in long reach PON and SDN-controlled core," in Proc. OFC, Los Angeles, USA, Mar. 2015.

[19] X. Dong, T. El-Gorashi, and J. M. H. Elmirghani, "IP over WDM networks employing renewable energy sources," IEEE/OSA JLT, vol. 29, no. 1, pp. 3-14, Jan. 2011.

[20] P. Ohlen, B. Skubic, A. Rostami, M. Fiorani, P. Monti, Z. Ghebretensae, J. Martensson, K. Wang, and L. Wosinska, "Data plane and control architectures for $5 \mathrm{~g}$ transport networks," Journal of Lightwave Technology, vol. 34, no. 6, pp. 15011508, March 2016.

[21] Jonas Weiss, Roger Dangel, Jens Hofrichter, Folkert Horst, Daniel Jubin, Norbert Meier, Antonio La Porta, and Bert Jan Offrein, "Optical interconnects for disaggregated resources in future datacenters," in Proc. ECOC, Cannes, France, Sep. 2014

[22] Martijn J. R. Heck, Jared F. Bauters, Michael L. Davenport, Jonathan K. Doylend, Siddharth Jain, Géza Kurczveil, Sudharsanan Srinivasan, Yongbo Tang, and John E. Bowers, "Hybrid silicon photonic integrated circuit technology," IEEE Journal of Selected Topics in Quantum Electronics, vol. 19, no. 4, pp. 6100117-6100117, July/Aug. 2013.

[23] J. Summers, T. Vallaitis, P. Evans, M. Ziari, P. Studenkov, M. Fisher, J. Sena, A. James, S. Corzine, D. Pavinski, J. OuYang, M. Missey, D. Gold, W. Williams, M. Lai, D. Welch, and F. Kish, "Monolithic inp-based coherent transmitter photonic integrated circuit with 2.25 tbit/s capacity," IET Electronics Letters, vol. 50, no. 16, pp. 1150-1152, July. 2014.

[24] Andy Eu-Jin Lim, Junfeng Song, Qing Fang, Chao Li, X.iaoguang Tu, Ning Duan, Kok Kiong Chen, Roger PohCher Tern, and Tsung-Yang Liow, "Review of silicon photonics foundry efforts," IEEE Journal of Selected Topics in Quantum Electronics, vol. 20, no. 4, pp. 405-416, July 2014.

[25] Cheng Wang, Neda Nasiriani, George Kesidis, Bhuvan Urgaonkar, Qian Wang, Lydia Y. Chen, Aayush Gupta, and
Robert Birke, "Recouping energy costs from cloud tenants: Tenant demand response aware pricing design," in ACM eEnergy, 2015, pp. 141-150.

[26] Chuangang Ren, Di Wang, Bhuvan Urgaonkar, and Anand Sivasubramaniam, "Carbon-aware energy capacity planning for datacenters," in IEEE MASCOTS, 2012, pp. 391-400.

[27] Zhenhua Liu, Adam Wierman, Yuan Chen, Benjamin Razon, and Niangjun Chen, "Data center demand response: Avoiding the coincident peak via workload shifting and local generation," Performance Evaluation, vol. 70, no. 10, pp. 770-791, 2013.

[28] Zhenhua Liu, Minghong Lin, Adam Wierman, Steven H. Low, and Lachlan L. H. Andrew, "Greening geographical load balancing," IEEE/ACM Trans. Netw., vol. 23, no. 2, pp. 657-671, 2015.

[29] James R. Hamilton, "An architecture for modular data centers," in CIDR, 2007, pp. 306-313.

[30] Iñigo Goiri, William A. Katsak, Kien Le, Thu D. Nguyen, and Ricardo Bianchini, "Parasol and greenswitch: managing datacenters powered by renewable energy," in ASPLOS, 2013, pp. 51-64.

[31] Ronald P. Luijten, Dae Pham, Rolf Clauberg, Matteo Cossale, Huy N. Nguyen, and Mihir Pandya, "4.4 energy-efficient microserver based on a 12 -core $1.8 \mathrm{ghz} 188 \mathrm{k}$-coremark $28 \mathrm{~nm}$ bulk CMOS $64 \mathrm{~b}$ soc for big-data applications with $159 \mathrm{gb} / \mathrm{s} / 1$ memory bandwidth system density," in IEEE ISSCC, 2015, pp. 1-3.

[32] Vasileios Lakafosis, Sreenivas Addagatla, Christian Belady, and Suyash Sinha, "Prometheus: A wirelessly interconnected, pico-datacenter framework for the developing world," in WWIC, 2012, pp. 26-39.

[33] Yijun Ying, Robert Birke, Cheng Wang, Lydia Y. Chen, and Natarajan Gautam, "Optimizing energy, locality and priority in a mapreduce cluster," in IEEE ICAC, 2015, pp. 21-30.

[34] Chih-Lin I, Corbett Rowell, Shuangfeng Han, Zhikun Xu, Gang Li, and Zhengang Pan, "Toward green and soft: a 5G perspective," IEEE Commun. Mag., vol. 52, no. 2, pp. 66-73, 2014.

[35] Aleksandra Checko, Henrik L Christiansen, Ying Yan, Lara Scolari, Georgios Kardaras, Michael S Berger, and Lars Dittmann, "Cloud RAN for mobile networks: a technology overview," IEEE Commun. Surveys Tuts., vol. 17, no. 1, pp. 405-426, 2015.

[36] Matteo Fiorani, Björn Skubic, Jonas Mårtensson, Luca Valcarenghi, Piero Castoldi, Lena Wosinska, and Paolo Monti, "On the design of 5g transport networks," Photonic Network Communications, vol. 30, no. 3, pp. 403-415, 2015.

[37] G. Bianchi, E. Biton, N. Blefari-Melazzi, I. Borges, L. Chiaraviglio, P. de la Cruz Ramos, P. Eardley, F. Fontes, M. J. McGrath, L. Natarianni, D. Niculescu, C. Parada, M. Popovici, V. Riccobene, S. Salsano, B. Sayadi, J. Thomson, C. Tselios, and G. Tsolis, "Superfluidity: a Flexible Functional Architecture for 5G Networks," Transactions on Emerging Telecommunication Technologies, on press 2016.

[38] Sibel Tombaz, Pal Frenger, Fredrik Athley, Eliane Semaan, Claes Tidestav, and Anders Furuskar, "Energy performance of 5G-NX wireless access utilizing massive beamforming and an ultra-lean system design," in IEEE GLOBECOM, December 2015, pp. 1-7. 